\title{
Seguimiento de pacientes con atipia de células escamosas de significado indeterminado (ASCUS) en un servicio citológico de Medellín (Colombia), 2011-2015
}

\author{
Monitoring of patients with atypical squamous cells \\ of undetermined significance (ASCUS) in a cytological \\ service from Medellin (Colombia), 2011-2015
}

\author{
Kelly J. Agudelo-Hincapié MB ${ }^{1}$, Diana L. García-Carvajal MB ${ }^{1}$, \\ Jaiberth A. Cardona-Arias MSc', Marleny Valencia-Arredondo PhD ${ }^{3}$
}

Introducción: en la literatura científica nacional existen pocos estudios sobre el seguimiento de mujeres con atipias de células escamosas de significado indeterminado (ASCUS). Objetivo: describir el seguimiento a cinco años de una cohorte retrospectiva de mujeres con resultado de ASCUS en un servicio citológico de Medellín, Colombia. Materiales y métodos: se realizó un estudio de una cohorte retrospectiva de mujeres con ASCUS en una Institución Prestadora de Servicios de Salud de Medellín, entre 2011 y 2015. Se evaluaron aspectos sociodemográficos, signos, síntomas y hallazgos preneoplásicos a partir de una fuente de información secundaria. Se determinó la frecuencia de mujeres sin control citológico, con regresión de la lesión, persistencia o progresión a lesión intraepitelial

\footnotetext{
${ }^{1}$ Estudiantes Microbiología y Bioanálisis, Escuela de Microbiología, Universidad de Antioquia. Medellín, Colombia.

2 Microbiólogo y Bioanalista, MSc en Epidemiología y Economía aplicada. Docente, Grupo de Investigación Salud y Sostenibilidad, Universidad de Antioquia y Facultad de Medicina, Universidad Cooperativa de Colombia. Medellín, Colombia.

${ }^{3}$ Bacterióloga y Laboratorista Clínica, Especialista en Citología Cervicouterina, MSc y PhD en Salud Pública. Docente, Grupo Salud Sexual y Cáncer, Escuela de Microbiología, Universidad de Antioquia. Medellín, Colombia. Correspondencia: Calle 67 N. ${ }^{\circ}$ 53-108, Bloque 5, oficina 410. Teléfono: 574 2195495. Fax: 574 2195486. Correo electrónico: marleny.valencia@udea.edu.co

Conflicto de intereses: los autores declaran que no tienen conflicto de intereses Medicina \& Laboratorio 2016; 22: 447-458

Módulo 19 (Investigación), número 48. Editora Médica Colombiana S.A. $2016^{\circ}$

Recibido el 13 de octubre de 2016; aceptado el 27 de octubre de 2016
} 
escamosa (LIE). Se estimó la proporción de incidencia de ASCUS y LIE en cada año de seguimiento. Resultados: se incluyeron 2.771 mujeres con diagnóstico de ASCUS. La incidencia de regresión al primer control fue 69,8\% y en el último $84,6 \%$ y de progresión $10,8 \%$ y 6,6\%, respectivamente, con menos del 1,0\% de progresión a LIE de alto grado. La incidencia de ASCUS osciló entre 3,6\% (2012) y $5,7 \%$ (2013) mientras que de LIE entre 1,1\% (2012) y 2,4\% (2014). El 28,1\% de la población nunca asistió a un control. Conclusiones: se halló una elevada frecuencia de regresión de las lesiones clasificadas como ASCUS y de mujeres sin control durante cinco años; esto evidencia la necesidad de mejorar las estrategias de seguimiento citológico que redunden en la disminución de costos asociados a la implementación de otras pruebas diagnósticas.

Palabras clave: lesiones intraepiteliales escamosas de cuello uterino, prueba de Papanicolaou, estudios de seguimiento.

Introduction: In the national scientific literature are few studies about the follow-up of women with atypical squamous cells of undetermined significance (ASCUS). Objective: To describe the monitoring of a retrospective cohort of women with ASCUS result in a cytological service of Medellin, Colombia. Materials and methods: A descriptive study of a retrospective cohort of women with an ASCUS diagnosis obtained in an institution of health from Medellin (Colombia), between 2011 and 2015 was performed. Socio-demographics, signs, symptoms and preneoplasic findings were evaluated from a secondary source of information. Frequency of women without cytological control, regression, progression, or persistence of lesions was determined. The incidence rate of ASCUS and squamous intraepithelial lesion (SIL) in each year of follow-up was estimated. Results: 2,771 women diagnosed with ASCUS were included. The incidence of regression in the first control was $69.8 \%$ and $84.7 \%$ in the last one and of progression was $10.8 \%$ and $6.6 \%$, respectively, with less than $1.0 \%$ of progression to high grade squamous intraepithelial lesion. The ASCUS incidence ranged from 3.6\% (2012) and 5.7\% (2013) while squamous intraepithelial lesion incidence was between 1.1\% (2012) and $2.4 \%$ (2014). Finally, $28.1 \%$ of the population never attended a control. Conclusions: A high frequency of regression of ASCUS lesions and a high proportion of women without control during 5 years was found; this shows the need to improve cytological control strategies and the costs associated with the implementation of other diagnostic tests.

Keywords: squamous intraepithelial lesions of the cervix, Papanicolaou test, follow-up studies.

Agudelo-Hincapié KJ, García-Carvajal DL, Cardona-Arias JA, Valencia-Arredondo M. Seguimiento de pacientes con atipia de células escamosas de significado indeterminado (ASCUS) en un servicio citológico de Medellín, 2011-2015. Medicina \& Laboratorio 2016; 22: 447-458. 
L as atipias de células escamosas de significado indeterminado (ASCUS) son definidas como cambios celulares anormales más marcados que un cambio reactivo o inflamatorio, que incluyen aumento del tamaño en el núcleo, leve aumento en la relación núcleo-citoplasma, binucleación, entre otras, y que no son indicadores de cambios reactivos ni cumplen con los criterios para el diagnóstico de lesión intraepitelial escamosa (LIE) [1]. El término ASCUS es introducido en 1988 por el sistema Bethesda, creado para estandarizar los conceptos para la descripción del reporte citológico. En su última actualización (2001), el sistema realiza la modificación más importante a la categoría ASCUS, la cual pasa a denominarse células escamosas atípicas (ASC), que se subdividen en células escamosas atípicas de significado indeterminado (ASCUS) y células escamosas atípicas no excluyente de lesión escamosa de alto grado (ASC-H) [2].

La categoría ASCUS, como tal, corresponde a una clasificación indeterminada en la que no se puede asegurar qué tipo de alteración se observa en la citología. Una publicación reciente sobre el sistema Bethesda para 2014 mostró cómo la introducción de ASCUS permitió agrupar a las pacientes cuyas citologías presentan limitaciones en su interpretación morfológica, lo que se deriva en un aumento en la frecuencia de las pacientes en esta categoría [3].

En la ciudad de Medellín (Colombia) la prevalencia de ASCUS ha oscilado entre 3,2\% y $4,8 \%$ en mujeres de población general atendidas en programas de control de cáncer de cuello uterino $[4,5]$. Otros estudios en Colombia reportan prevalencias entre el $2,5 \%$ y $10 \%[6,7]$, mientras que investigaciones de otros países han reportado frecuencias relativas del $7 \%$ [8]. En general, se acepta que la tasa ideal de estas alteraciones no debe superar el $5 \%$ de los reportes de citologías alteradas [9].

La existencia de ASCUS puede abarcar tanto procesos inflamatorios con un curso benigno así como alteraciones que posteriormente pueden derivar en lesiones intraepiteliales de bajo y alto grado e incluso invasivas, lo que representa un mayor riesgo de lesiones en mujeres con ASCUS en comparación con la población general que justifica una mayor exhaustividad en su seguimiento [10]. En este sentido, en Colombia se recomiendan tres protocolos diferentes en mujeres con ASCUS: a) seguimiento citológico, b) pruebas de detección del VPH y c) colposcopia o biopsia. No obstante, no existe un consenso claro sobre el tipo de prueba o seguimiento que entregue los mejores resultados para los diferentes grupos poblacionales, lo que lleva a la desorientación en la conducta clínica, los resultados desfavorables en el seguimiento de las pacientes y el incremento de los costos para el sistema de salud [11].

Algunos estudios previos han indicado que las alteraciones tipo ASCUS pueden regresar espontáneamente, caso en el cual el control citológico semestral o anual constituiría una medida suficiente para el seguimiento. Esta determinación se establece con base en las estimaciones que indican que el riesgo de tener cáncer para este grupo se encuentra entre $0,1 \%$ y $0,2 \%[12,13]$. Otras opciones de seguimiento 
de estas lesiones incluyen la citología de base líquida, pero esta resulta más costosa y complicada que la convencional y no registra diferencias significativas en el diagnóstico de una lesión intraepitelial escamosa ni de carcinoma invasor. Por su parte, la detección de ADN del virus del papiloma humano (VPH) presenta baja utilidad en el seguimiento de las pacientes adolescentes o adultas jóvenes sexualmente activas por varias razones: a) en esta etapa de la vida es alta la prevalencia de la infección, b) la variabilidad en el riesgo de displasia y c) la baja frecuencia de carcinoma (menor al $0,1 \%$ antes de los 21 años) [14].

En el seguimiento de las mujeres con ASCUS a través de la citología convencional se ha reportado una frecuencia de progresión a lesión intraepitelial escamosa de alto grado del $5 \%$ al $17 \%$. El riesgo aumenta hasta un $17,7 \%$ en la evaluación inicial si la paciente es positiva para genotipos de alto riesgo del virus y a un $8,8 \%$ más durante un periodo de seguimiento de dos años. Asimismo, se considera que las ASCUS con persistencia del ADN viral por más de 12 a 18 meses es predictor de riesgo de futuras lesiones intraepiteliales, especialmente en el caso de infecciones por los genotipos 16 y $18 \mathrm{del} \mathrm{VPH}$. Contario a esto, otros autores indican que, a pesar de la elevada exposición al VPH (cerca del $60 \%$ de mujeres) por inicio de las relaciones sexuales durante la adolescencia, la mayoría de mujeres desarrollan inmunidad natural que autolimita la infección y deriva en la regresión de las alteraciones en aproximadamente 24 meses [15].

En Chile se realizó el seguimiento citológico de ASCUS en 154 mujeres en el que se encontró una evolución de las citologías atípicas a lesiones de alto grado en 21,4\% casos y a carcinomas invasores en un $1,3 \%$ [16]. Otro estudio publicado por el Ministerio de Salud de Chile mostró que entre el $5 \%$ y el $17 \%$ de las mujeres con citología atípica pueden tener una lesión tipo neoplasia intraepitelial cervical (NIC) 2 o 3 confirmado por biopsia y que esto aumenta entre un $24 \%$ y $94 \%$ en las pacientes con reporte de ASC-H que no descarta lesión de alto grado [17].

Los antecedentes expuestos muestran una amplia variedad en las formas de evaluar la evolución de las lesiones clasificadas citológicamente como ASCUS así como la frecuencia de progresión o regresión, al tiempo que demuestra una baja disponibilidad de estudios en Colombia al respecto. Por ello, el objetivo de este estudio fue describir el seguimiento de una cohorte retrospectiva de mujeres con resultado de ASCUS en una Institución Prestadora de Servicios de Salud de Medellín en el periodo de 2011 a 2015.

\section{Materiales y métodos}

\section{Tipo y población de estudio}

Se realizó un estudio descriptivo de una cohorte retrospectiva de mujeres atendidas en el Servicio de Citología de la Escuela de Microbiología de la Universidad de Antioquia (Medellín, Colombia) entre 2011 y 2015. Se incluyeron las pacientes 
con reporte citológico de ASCUS en el 2011 y se excluyeron aquellas con registros de muestras con calidad insatisfactoria y procedimientos previos al ASCUS tales como conización, radioterapia y quimioterapia.

\section{Recolección de la información y control de sesgos}

Como fuente de información se utilizó la base de datos del Servicio de Citología de la Escuela de Microbiología de la Universidad de Antioquia, de la cual se obtuvieron las variables relacionadas con la edad, sector de procedencia, hallazgos microbiológicos, hallazgos oncológicos como lesión intraepitelial escamosa de alto y bajo grado, hallazgos de significado indeterminado como células escamosas atípicas de significado indeterminado (ASCUS), células escamosas atípicas no excluyente de lesión escamosa de alto grado (ASC-H), células glandulares atípicas (ACG) y células glandulares atípicas-otras no especificadas (ACG-NOS) a favor de neoplasia, y otros reportes como inflamación, atrofia, reparación y radiación.

Para el control de sesgos de información se evaluó la reproducibilidad intra e interobservador de la extracción de la información de un semestre en dos ocasiones diferentes.

\section{Análisis estadístico}

La población de estudio se describió con medidas de resumen para la edad y frecuencias para la zona de procedencia, signos, síntomas y hallazgos microbiológicos. Para cada uno de los cinco años de seguimiento (2011 a 2015) se determinó la prevalencia de mujeres sin control citológico e incidencia de regresión de la alteración indeterminada (ASCUS), así como su persistencia o progresión a una lesión intraepitelial, todas con su intervalo de confianza.

A partir de las mujeres que presentaron regresión de las alteraciones tipo ASCUS en uno de los controles citológicos se estimó la proporción de incidencia de ASCUS y de lesión intraepitelial escamosa en el control del año siguiente. Se presentó el seguimiento a tres años (2011 al 2013) de las mujeres en cada uno de las categorías o grupos de estudio analizados (sin control citológico, con regresión, persistencia o progresión de la alteración tipo ASCUS) durante el primer control del 2011; además, se describió la proporción de mujeres que se mantuvieron en el mismo grupo o categoría de estudio durante los controles realizados en los cinco años de estudio. Los análisis se realizaron en el programa estadístico SPSS versión 24.0 (IBM, Nueva York, Estados Unidos), con una significación del 0,05.

\section{Aspectos éticos}

En todas las etapas del proyecto se tuvieron presentes los principios de la resolución 008430 de 1993 del Ministerio de Salud de la República de Colombia que clasifica este estudio como una investigación sin riesgo. Además, en los datos que se tomaron para este estudio se garantizó la confidencialidad de la información y el acceso a la base de datos fue exclusivo para los investigadores del estudio. 


\section{Resultados}

\section{Características de la población de estudio}

Se incluyeron un total de 2.771 pacientes con reporte citológico de ASCUS en el 2011 y fueron excluidos 3.389 pacientes con muestras con calidad insatisfactoria y procedimientos como conización, radioterapia y quimioterapia previos al ASCUS.

La edad media de las mujeres incluidas fue $34,0 \pm 13,2$, de las cuales el $50 \%$ tenía 32 años o más; el rango intercuartílico osciló entre 22 y 44 años y el rango entre 13 y 81 . La mayor proporción de pacientes correspondió al grupo de edad entre 21 y 30 años, residentes en la zona Nororiental de la ciudad de Medellín (Colombia), que presentaban síntomas como amenorrea y prurito (véase tabla 1 ).

En 2011 la frecuencia de mujeres sin el primer control citológico fue del $81,3 \%$, la regresión de la alteración tipo ASCUS en el primer control fue del $13,0 \%$, la persistencia del 3,6\% y la progresión a lesión intraepitelial escamosa del 2,0\% (de estas el $0,2 \%$ correspondieron a lesiones de

\begin{tabular}{|c|c|c|c|}
\hline \multicolumn{4}{|c|}{$\begin{array}{l}\text { Tabla 1. Descripción del grupo etario, la zona } \\
\text { de procedencia y algunas características clí- } \\
\text { nicas en la población de estudio }(n=2.771)\end{array}$} \\
\hline Parámetro & & $\begin{array}{l}\text { Frecuencia } \\
\text { absoluta } \\
\left(\mathrm{N} .{ }^{\circ}\right)\end{array}$ & $\begin{array}{l}\text { Frecuencia } \\
\text { relativa } \\
(\%)\end{array}$ \\
\hline \multirow[t]{8}{*}{ Zona } & Sin dato & 90 & 3,2 \\
\hline & Nororiental & 970 & 35,0 \\
\hline & Noroccidental & 577 & 20,8 \\
\hline & Centroriental & 306 & 11,0 \\
\hline & Centroccidental & 385 & 13,9 \\
\hline & Suroriental & 2 & 0,1 \\
\hline & Suroccidental & 209 & 7,5 \\
\hline & Rural & 232 & 8,4 \\
\hline \multirow{5}{*}{$\begin{array}{l}\text { Grupo } \\
\text { etario* }\end{array}$} & Adolescentes & 502 & 18,1 \\
\hline & 21-30 años & 799 & 28,8 \\
\hline & $31-40$ años & 564 & 20,4 \\
\hline & 41-50 años & 580 & 20,9 \\
\hline & $\begin{array}{l}\text { Mayores } \\
\text { de } 50 \text { años }\end{array}$ & 325 & 11,7 \\
\hline \multirow{8}{*}{$\begin{array}{l}\text { Signos y } \\
\text { síntomas** }\end{array}$} & Amenorrea & 1095 & 39,5 \\
\hline & Prurito & 1040 & 37,5 \\
\hline & Ardor & 628 & 22,7 \\
\hline & Dispareunia & 457 & 16,5 \\
\hline & Flujo & 43 & 1,6 \\
\hline & $\begin{array}{l}\text { Lesión en } \\
\text { genitales }\end{array}$ & 348 & 12,6 \\
\hline & $\begin{array}{l}\text { Sangrado } \\
\text { anormal }\end{array}$ & 266 & 9,6 \\
\hline & $\begin{array}{l}\text { Infección } \\
\text { urinaria }\end{array}$ & 93 & 3,4 \\
\hline \multicolumn{4}{|c|}{$\begin{array}{l}\text { *No se halló registro de la edad en una paciente } \\
\text { **Algunas pacientes presentaban más de un signo o síntoma } \\
\text { simultáneamente }\end{array}$} \\
\hline
\end{tabular}
alto grado) (véase tabla 2). Al excluir del análisis a las mujeres sin control citológico en cada uno de los años de estudio se encontró una frecuencia de regresión de ASCUS del 69,8\% (361/517) en 2011, 80,4\% (1.007/1.252) en 2012, $80,4 \%(822 / 1.023)$ en $2013,79,0 \%(654 / 828)$ en 2014 y $84,6 \%(643 / 760)$ en 2015; mientras que de progresión a lesión intraepitelial escamosa fue de 10,8\% (56/517), 6,6\% (83/1.252), 6,7\% (69/1.023), 7,0\% (58/828) y 6,6\% (50/760), respectivamente; siendo más elevada la frecuencia de lesión intraepitelial escamosa de bajo grado, entre 5,2\% (53/1.023) en el 2013 y 9,8\% (50/517) en el 
2011 , respecto a las de alto grado que fluctuaron entre $0,8 \%(10 / 1.252)$ en el 2012 y $1,5 \%(12 / 828)$ en el 2014.

De las alteraciones que progresaron en 2013 el 0,5\% fue a lesión intraepitelial escamosa de alto grado, mientras que en 2014 y 2015 la prevalencia de este tipo de lesión correspondió al 0,1\% y el 0,0\%, respectivamente. Por su parte, en la incidencia acumulada de las alteraciones se encontraron ASCUS en un $3,6 \%$ (13/361) en 2012, 5,8\% (58/1.008) en 2013, 5,6\% (46/822) en 2014 y 3,4\% (21/624) en 2015 ; mientras que la incidencia de la lesión intraepitelial escamosa fue de $1,1 \%$ (4/361), $1,5 \%$ (15/1.008), 2,4\% (20/822) y $2,1 \%(13 / 624)$ en los mismos años (véase tabla 2).

\begin{tabular}{|c|c|c|c|c|c|c|c|c|c|c|}
\hline \multirow[t]{2}{*}{ Característica } & \multicolumn{2}{|c|}{2011} & \multicolumn{2}{|c|}{2012} & \multicolumn{2}{|c|}{2013} & \multicolumn{2}{|c|}{2014} & \multicolumn{2}{|c|}{2015} \\
\hline & N. ${ }^{\circ}$ & $\begin{array}{l}\%(I C \\
95 \%)\end{array}$ & N. ${ }^{\circ}$ & $\begin{array}{l}\%(I C \\
95 \%)\end{array}$ & N. ${ }^{\circ}$ & $\begin{array}{l}\%(I C \\
95 \%)\end{array}$ & N. ${ }^{\circ}$ & $\begin{array}{l}\% \text { (IC } \\
95 \%)\end{array}$ & N. ${ }^{\circ}$ & $\begin{array}{l}\% \text { (IC } \\
95 \%)\end{array}$ \\
\hline $\begin{array}{l}\text { Ausencia } \\
\text { de control }\end{array}$ & 2.254 & $\begin{array}{l}81,3 \\
(79,9- \\
82,8)\end{array}$ & 1.519 & $\begin{array}{l}54,8 \\
(52,9- \\
56,6)\end{array}$ & 1.748 & $\begin{array}{l}63,1 \\
(61,3- \\
64,9)\end{array}$ & 1.942 & $\begin{array}{l}70,1 \\
(68,3- \\
71,8)\end{array}$ & 2.012 & $\begin{array}{l}72,6 \\
(70,9- \\
74,3)\end{array}$ \\
\hline Regresión & 361 & $\begin{array}{l}13,0 \\
(11,8- \\
14,3)\end{array}$ & 1.007 & $\begin{array}{l}36,3 \\
(34,6- \\
38,2)\end{array}$ & 822 & $\begin{array}{l}29,7 \\
(27,9- \\
31,4)\end{array}$ & 654 & $\begin{array}{l}23,6 \\
(22,0- \\
25,2)\end{array}$ & 643 & $\begin{array}{l}23,2 \\
(21,6- \\
24,8)\end{array}$ \\
\hline Persistencia & 100 & $\begin{array}{l}3,6 \\
(2,9- \\
4,3)\end{array}$ & 162 & $\begin{array}{l}5,8 \\
(5,0- \\
6,7)\end{array}$ & 132 & $\begin{array}{l}4,8 \\
(4,0- \\
5,6)\end{array}$ & 116 & $\begin{array}{l}4,2 \\
(3,5- \\
5,0)\end{array}$ & 67 & $\begin{array}{l}2,4 \\
(1,8- \\
3,0)\end{array}$ \\
\hline Progresión & 56 & $\begin{array}{l}2,0 \\
(1,5- \\
2,6)\end{array}$ & 83 & $\begin{array}{l}3,0 \\
(2,3- \\
3,6)\end{array}$ & 69 & $\begin{array}{l}2,5 \\
(1,9- \\
3,0)\end{array}$ & 58 & $\begin{array}{l}2,1 \\
(1,6- \\
2,7)\end{array}$ & 50 & $\begin{array}{l}1,8 \\
(1,3- \\
2,4)\end{array}$ \\
\hline \multicolumn{11}{|c|}{ Incidencia acumulada de alteraciones } \\
\hline ASCUS & -- & -- & 13 & $\begin{array}{l}3,6 \\
(1,5- \\
5,7)\end{array}$ & 58 & $\begin{array}{l}5,8 \\
(4,3- \\
7,2)\end{array}$ & 46 & $\begin{array}{l}5,6 \\
(4,0- \\
7,2)\end{array}$ & 21 & $\begin{array}{l}3,4 \\
(1,9- \\
4,9)\end{array}$ \\
\hline $\begin{array}{l}\text { Lesión in- } \\
\text { traepitelial } \\
\text { escamosa }\end{array}$ & -- & -- & 4 & $\begin{array}{l}1,1 \\
(0,3- \\
2,8)\end{array}$ & 15 & $\begin{array}{l}1,5 \\
(0,7- \\
2,3)\end{array}$ & 20 & $\begin{array}{l}2,4 \\
(1,3- \\
3,5)\end{array}$ & 13 & $\begin{array}{l}2,1 \\
(0,9- \\
3,3)\end{array}$ \\
\hline
\end{tabular}

En la tabla 3 se presenta el recuento de mujeres en cada una de las categorías diagnósticas analizadas durante el primer control en el 2011 y sus resultados de seguimiento en los dos años siguientes. Entre los resultados se destacan los siguientes: a) de las 2.254 mujeres que no se hicieron el control en el 2011 para el 2012 el 35,3\% ( $n=796)$ presentó regresión de la alteración (resultado negativo) y el 3,1\% ( $n=70)$ progresión, b) de las 361 mujeres con regresión en el primer control (2011) el 3,6\% ( $n=13)$ volvió a registrar ASCUS y el $1,1 \%(n=4)$ una lesión intraepitelial escamosa y c) de las 100 mujeres en las que persistía la alteración en 2011 el 34,0\% $(n=34)$ presentó regresión y el 3,0\% $(n=3)$ progresión a lesión intraepitelial escamosa. 


\begin{tabular}{|c|c|c|c|c|c|c|c|c|c|}
\hline \multirow[t]{3}{*}{ Seguimiento 2012} & \multirow{3}{*}{$\begin{array}{l}\text { Seguimiento } \\
2013\end{array}$} & \multicolumn{8}{|c|}{ Seguimiento 2011} \\
\hline & & \multicolumn{2}{|c|}{$\begin{array}{l}\text { Sin control } \\
(n=2.254)\end{array}$} & \multicolumn{2}{|c|}{$\begin{array}{l}\text { Regresión } \\
(n=361)\end{array}$} & \multicolumn{2}{|c|}{$\begin{array}{l}\text { Persistencia } \\
(n=100)\end{array}$} & \multicolumn{2}{|c|}{$\begin{array}{l}\text { Progresión } \\
(n=56)\end{array}$} \\
\hline & & N. ${ }^{\circ}$ & $\%$ & N. ${ }^{\circ}$ & $\%$ & N. ${ }^{\circ}$ & $\%$ & N. ${ }^{\circ}$ & $\%$ \\
\hline \multirow{5}{*}{$\begin{array}{l}\text { Sin control } \\
(n=1.518)\end{array}$} & Sin control & 937 & 41,6 & 123 & 34,1 & 34 & 34,0 & 19 & 33,9 \\
\hline & Negativa & 255 & 11,3 & 46 & 12,7 & 17 & 17,0 & 10 & 17,9 \\
\hline & ASCUS & 32 & 1,4 & 9 & 2,5 & 3 & 3,0 & 0 & 0,0 \\
\hline & LIE & 22 & 1,0 & 4 & 1,1 & 4 & 4,0 & 3 & 5,4 \\
\hline & Subtotal & 1.246 & 55,3 & 182 & 50,4 & 58 & 58,0 & 32 & 57,1 \\
\hline \multirow{5}{*}{$\begin{array}{l}\text { Negativas } \\
(n=1.008)\end{array}$} & Sin control & 429 & 19,0 & 72 & 19,9 & 10 & 10,0 & 2 & 3,6 \\
\hline & Negativa & 311 & 13,8 & 78 & 21,6 & 22 & 22,0 & 11 & 19,6 \\
\hline & ASCUS & 46 & 2,0 & 7 & 1,9 & 2 & 2,0 & 3 & 5,4 \\
\hline & LIE & 10 & 0,4 & 5 & 1,4 & 0 & 0,0 & 0 & 0,0 \\
\hline & Subtotal & 796 & 35,3 & 162 & 44,9 & 34 & 34,0 & 16 & 28,6 \\
\hline \multirow{5}{*}{$\begin{array}{l}\text { Alteración de signifi- } \\
\text { cado indeterminado } \\
(n=162)\end{array}$} & Sin control & 74 & 3,3 & 5 & 1,4 & 3 & 3,0 & 2 & 3,6 \\
\hline & Negativa & 43 & 1,9 & 6 & 1,7 & 0 & 0,0 & 0 & 0,0 \\
\hline & ASCUS & 18 & 0,8 & 1 & 0,3 & 1 & 1,0 & 0 & 0,0 \\
\hline & LIE & 7 & 0,3 & 1 & 0,3 & 1 & 1,0 & 0 & 0,0 \\
\hline & Subtotal & 142 & 6,3 & 13 & 3,6 & 5 & 5,0 & 2 & 3,6 \\
\hline \multirow{5}{*}{$\begin{array}{l}\text { Lesión intraepitelial } \\
\text { escamosa (LIE) } \\
(n=83)\end{array}$} & Sin control & 36 & 1,6 & 1 & 0,3 & 0 & 0,0 & 1 & 1,8 \\
\hline & Negativa & 17 & 0,8 & 2 & 0,6 & 1 & 1,0 & 3 & 5,4 \\
\hline & ASCUS & 11 & 0,5 & 0 & 0,0 & 0 & 0,0 & 0 & 0,0 \\
\hline & LIE & 6 & 0,3 & 1 & 0,3 & 2 & 2,0 & 2 & 3,6 \\
\hline & Subtotal & 70 & 3,1 & 4 & 1,1 & 3 & 3,0 & 6 & 10,7 \\
\hline
\end{tabular}

Finalmente, en la tabla 4 se presenta la distribución porcentual de mujeres que no cambió la categoría diagnóstica inicial durante el seguimiento de cinco años, donde se destaca un $28,1 \%$ que nunca asistió a un control en el periodo estudiado y que en el $0,0 \%$ la alteración persistió o progresó a partir del tercer año de control.

\section{Discusión}

Las variaciones en la prevalencia de regresión espontánea de las alteraciones citológicas tipo ASCUS en los diferentes años de control del presente estudio $(69,8 \%$ en el primero y $84,6 \%$ en el último) al igual que de progresión a lesiones intraepiteliales escamosas ( $10,8 \%$ en el primer control y $6,6 \%$ en el último del año 2015$)$ demuestran la heterogeneidad en las proporciones de ambos procesos en la población con lesiones ASCUS, al tiempo que manifiestan la necesidad de mejorar el seguimiento de este grupo como base para orientar acciones preventivas.

La distribución de ASCUS se presentó con mayor frecuencia en las mujeres entre los 21 y los 30 años, lo cual coincide con otros estudios realizados en la ciudad de 


\begin{tabular}{|c|c|c|c|c|c|}
\hline \multirow[t]{2}{*}{ Categoría } & \multicolumn{5}{|c|}{ N. ${ }^{\circ}(\%)$} \\
\hline & 2011 & 2012 & 2013 & 2014 & 2015 \\
\hline Sin control & $2.254(81,3)$ & $1.246(45,0)$ & $937(33,8)$ & $842(30,4)$ & $778(28,1)$ \\
\hline Regresión & $361(13,0)$ & $162(5,8)$ & $78(2,8)$ & $39(1,4)$ & $19(0,7)$ \\
\hline Persistencia & $100(3,6)$ & $5(0,2)$ & $1(0,0)$ & $1(0,0)$ & $0(0,0)$ \\
\hline Progresión & $56(2,0)$ & $6(0,2)$ & $2(0,1)$ & $1(0,0)$ & $1(0,0)$ \\
\hline
\end{tabular}

Medellín (Colombia), donde, al igual que en el presente estudio, la prevalencia de lesiones intraepiteliales escamosas y ASCUS se encontró principalmente en este grupo poblacional [5]. Asimismo, en Venezuela se reportó un grupo de edad similar (entre los 20 y 24 años) como el de mayor ocurrencia de este tipo de alteraciones [18]. Clínica y epidemiológicamente esto podría explicarse por la elevada prevalencia de la infección por el virus del papiloma humano, particularmente de los subtipos 16 y 18, la susceptibilidad del aparato genital en la adolescencia y otros factores biológicos que contribuyen al desarrollo de la infección como extensa ectopia cervical, y la metaplasia escamosa activa, junto al elevado riesgo sexual por el inicio temprano de las relaciones sexuales, las múltiples parejas sin protección y el tabaquismo, que son frecuentes en este grupo poblacional [19].

Vale destacar que en este estudio el $28,1 \%$ de las mujeres nunca se hizo un control en el periodo de estudio de cinco años, lo que pondría de manifiesto la persistencia de limitaciones en el control del cáncer cervicouterino, debido, en muchas ocasiones, al cambio en el régimen de afiliación en salud de las usuarias del programa. Estos datos concuerdan con el estudio de Wiesner y colaboradores (2010) [20], donde el 27\% de las mujeres con lesiones de alto grado o invasoras no tuvieron acceso a ninguno de los servicios diagnósticos o terapéuticos, mientras que en México se registró abandono de la atención médica en el 20,7\% de las mujeres con lesiones cervicales [21].

Múltiples condiciones podrían explicar el abandono del seguimiento en mujeres con esta alteración en Colombia, los cuales pueden vincularse con la administración de los servicios de salud, la atención clínica, los aspectos culturales, la vinculación de las mujeres a las actividades económicas que derivan en cambios en el tipo de afiliación en salud e institución prestadora de servicios de salud, la desarticulación de la atención inicial y la remisión a exámenes especializados, el seguimiento de las alteraciones y lesiones que no es obligatoria y, en algunos casos, la ausencia de un protocolo de manejo claro [20].

En este trabajo la frecuencia de regresión de las lesiones ASCUS osciló entre el $69,8 \%$ y el $84,6 \%$, lo que coincide con la prevalencia reportada por López-Alegría y colaboradores (2015) [22], en el que la tasa de regresión a la normalidad fue del 
$85,1 \%$, pero difiere de otro estudio publicado años antes (2011) por la misma autora donde el porcentaje de regresión fue del 56,5\% [16]. La no obtención de un 100\% de regresión podría ser sustentado en el hecho de que en la mayoría de mujeres, particularmente las jóvenes, la infección, e incluso la displasia, puede desaparecer por la acción del sistema inmune; no obstante, en proporciones mínimas, en las expuestas a subtipos de alto riesgo, la infección progresa a displasia o persiste aumentado el riesgo de padecer cáncer [23]. En coherencia con lo anterior, ante un primer reporte de ASCUS es recomendable realizar un segundo control citológico a los seis meses, ya que realizar una colposcopia, biopsia, escisión o ablación de la zona de transformación podría ser considerada una intervención excesiva que conduce a implicaciones como partos prematuros y genera costos adicionales [13].

Otros hallazgos del presente estudio indicaron una progresión a lesión intraepitelial escamosa que osciló entre el 6,6\% y el 10,8\%, para las lesiones intraepiteliales escamosas de alto grado las frecuencias se encontraron entre $0,8 \%$ y $1,5 \%$, mientras que para lesiones intraepiteliales escamosas de bajo grado entre $5,2 \%$ y $9,8 \%$. Lo anterior difiere con estudios de seguimiento en Santiago de Chile donde se reportan tasas más bajas de las lesiones de bajo grado $(3,6 \%)$ y mayores de las de alto grado $(11,6 \%)$ [22]. En otro estudio en 154 mujeres de Chile, en un periodo de tres años de seguimiento citológico e histológico, se encontraron $1,3 \%$ casos de carcinoma invasivo, $21,4 \%$ de lesiones intraepiteliales escamosas de bajo grado y $20,8 \%$ de lesiones intraepiteliales escamosas de alto grado [16].

La variabilidad expuesta entre los resultados del presente trabajo y los realizados en otros países puede explicarse por el tipo de métodos diagnósticos usados, ya que en este estudio se realizó sólo control citológico sin inclusión de datos relacionados con los resultados de colposcopia y biopsia los cuales pueden aumentar la eficiencia de la tamización citológica [22]. Sin embargo, se debe precisar que se aplicó lo indicado en la guía para el manejo de citologías atípicas en Colombia, en la que el manejo recomendado, en ausencia de la prueba de detección del virus del papiloma humano, es la repetición de la citología a los seis y a los doce meses o la realización de colposcopia inmediatamente; ante la persistencia de ASCUS en cualquiera de las dos citologías de seguimiento se debe remitir a la paciente a colposcopia y, en caso de regresión de la alteración, la mujer debe volver al programa de tamización de rutina [11].

Respecto a la incidencia acumulada de ASCUS por año en la población de estudio se encontró que osciló entre 3,3\% y 5,7\%. No existen otros estudios con los cuales se pueda correlacionar esta información debido a que la medida de ocurrencia que más se evalúa es la prevalencia, la cual indica la proporción de la población que padece la enfermedad en estudio en un momento dado y no la incidencia. A diferencia de los estudios de prevalencia, los estudios de incidencia inician con poblaciones susceptibles libres del evento en las que se observa la presentación de nuevos casos a lo largo de un periodo de seguimiento; de esta manera, los resultados no sólo indican el 
volumen final de casos nuevos aparecidos durante el seguimiento, sino que permiten definir en alguna medida la "velocidad" o frecuencia con la cual aparecen los casos en la población de referencia [24].

Por su parte, la incidencia acumulada de lesiones intraepiteliales escamosas de bajo y alto grado en la población estudiada por año osciló entre $1,1 \%$ y $2,4 \%$, lo cual difiere de lo encontrado por Sanabria-Negrin y colaboradores (2014) [25] en su estudio sobre incidencia de lesiones intraepiteliales escamosas que oscilaron entre $1,6 \%$ y $8,8 \%$. Sin embargo, son pocos los trabajos publicados que evalúan estos hallazgos, por lo que se infiere que las diferencias mostradas lograrían explicarse por las características de base de la población de estudio.

Entre las limitaciones del estudio se encuentra el hecho de no conocer las razones por las cuales una proporción importante de la población no se realizó control en los cinco años de seguimiento, por lo que no se puede establecer si este hecho es una limitación del programa o un problema estructural de la información de eventos en salud de Colombia donde el cambio de la institución prestadora del servicio de salud limita el seguimiento de las mujeres con alteraciones o lesiones.

Finalmente, se debe tener presente que la citología cervicouterina no presenta una sensibilidad del $100 \%$ y en algunos laboratorios puede registrar una moderada reproducibilidad, lo que puede derivar en una subestimación de la regresión o progresión de las lesiones tipo ASCUS; frente a esta limitante se sugiere que en estudios posteriores se compare la regresión o progresión con base en los resultados de la biopsia y no en la clasificación citológica. No obstante, vale la pena aclarar que en el laboratorio en que se desarrolló el presente estudio la validez y la reproducibilidad del estudio citológico se garantizaron mediante procesos de relectura de los reportes realizados por los citotecnólogos por parte de dos especialistas en citología y un patólogo, a lo que se suman otras acciones de control de calidad interno y externo de la IPS Universitaria.

\section{Conclusiones}

Se halló una elevada frecuencia de regresión de las lesiones citológicas clasificadas como ASCUS y una elevada proporción de mujeres sin control durante cinco años, que evidencia la necesidad de mejorar las estrategias de seguimiento citológico que redunden en la disminución de costos asociados a la implementación de otras pruebas diagnósticas.

\section{Agradecimientos}

Al Servicio de Citología de la Escuela de Microbiología de la Universidad de Antioquia (Medellín, Colombia). 


\section{Bibliografía}

1. García-Perlaza C, Amaya-Guío J, Naranjo E. Prevalencia de lesión escamosa intraepitelial de cérvix en pacientes con diagnóstico citológico de atipia escamosa de significado indeterminado (ASCUS), en una institución de complejidad media en Bogotá, Colombia. Rev Colomb Obstet Ginecol 2007; 58: $124-128$

2. López-Alegría F, Arcos E, González L, Soares De Lorenzi DR, Quezada O. Algoritmos de derivación y confirmación diagnóstica de citología cervical atípica: desafíos para la actualización. Rev Chil Obstet Ginecol 2012; 77: 322-328.

3. Nayar R, Wilbur DC. The Pap Test and Bethesda 2014. "The reports of my demise have been greatly exaggerated." (after a quotation from Mark Twain). Acta Cytol 2015; 59: 121-132.

4. Cardona-Arias JA, Valencia-Arredondo $M$. Prevalencia de alteraciones oncológicas en la $\mathrm{ci}$ tología cervicovaginal de pacientes de una institución prestadora de servicios de salud de Medellín 2010-2012. CES Med 2014; 28: 7-20.

5. Sampedro-Ossa CA, Ríos-Osorio LA, Cardona-Arias JA. Prevalencia de alteraciones preneoplásicas del cáncer de cuello uterino en un municipio del Norte de Antioquia-Colombia, 20082012. Archivos de Medicina 2014; 10: 1-10.

6. Mendoza T LA, Pedroza $\mathbf{P} \mathbf{M J}$, Micolta $\mathbf{C} \mathbf{P H}$ Ramirez R A, Cáceres G CR, López S DV, et al. Prevalencia de lesiones de bajo y alto grado de cuello uterino en una ciudad colombiana. Rev Chil Obstet Ginecol 2012; 77: 129-136.

7. Grisales H, Vanegas ÁP, Gaviria ÁM, Castaño J, Mora MA, Borrero M, et al. Prevalencia de anormalidades de células epiteliales y factoresasociados en mujeres de un municipio rural colombiano. Biomédica 2008; 28: 181-182.

8. Emerson RE, Puzanov A, Brunnemer C, Younger C, Cramer H. Long-term follow-up of women with atypical squamous cells of undetermined significance (ASCUS). Diagn Cytopathol 2002; 27: 153-157.

9. Muñiz-Fernandez N, Fernandez-Fernandez E, Escobar-Stein J, González-Vázquez LO Revisión de la categoría citológica "ASCUS"en el Hospital de Jove. VII Congreso Virtual Hispanoamericano de Anatomía Patológica y I Congreso de Preparaciones Virtuales por Internet. España: Oct $1-31 ; 2005$.

10. Picconi MA. Detección de virus papiloma humano en la prevención del cáncer cérvico-uterino. Medicina (B Aires) 2013; 73: 585-596.

11. República de Colombia, Ministerio de Salud y Protección Social, Instituto Nacional de Cancerología. Recomendaciones para el tratamiento de las pacientes con citología reportada con células escamosas atípicas de significado indeterminado (ASC-US) en Colombia. Bogotá D.C., Colombia. 2007. Disponible: http://medicina.udea.edu.co/Dependencias/Ginecologia/CLAP/ pdf/jornadas/2ascus2.pdf. Consultado: jun 2016.

12. República de Colombia, Ministerio de Salud y Protección Social, Dirección General de Promoción y Prevención. Norma técnica para la detección temprana del cáncer de cuello uterino y guía de atención de lesiones preneoplásicas de cuello uterino. Bogotá D.C., Colombia: Imprenta Nacional de Colombia. 2000.

13. Vega-Ulate GA. Prevencion de cancer cervical. Rev Méd Costa Rica Centroamérica 2013; LXX: 377-384.

14. Espinosa-Romero R, Arreola-Rosales RL, Velázquez-Hernández $\mathbf{N}$, Rodríguez-Reyes ER. Métodos de detección oportuna del cáncer cervicouterino. GAMO 2014; 13 (Supl. 4): 48-52.

15. Nazzal N O, Suárez P E, Larraguibel P R, Rojas $\mathbf{F ~ L , ~ B r o n d a ~} \mathbf{M} \mathbf{A}$. Lesiones preinvasoras de cuello uterino: Una visión actual. Rev Chil Obstet Ginecol 2006; 71: 341-348.

16. López F, Quezada O, Barrios T, López E. Follow-up of women with ASCUS in Chile. Diagn Cytopathol 2011; 39: 258-263.

17. República de Chile, Ministerio de Salud. Guía Clínica: Cáncer Cervicouterino. Santiago, Chile: Minsal. 2010. Disponible: http://www.minsal.cl/ portal/url/item/720bfefe91e9d2ede04001011f01 0ff2.pdf. Consultado: jun 2016.

18. Agüero A, Castillo K, González Blanco M. Neoplasia intraepitelial cervical de alto grado en mujeres menores de 25 años y mayores de 45 años. Rev Obstet Ginecol Venez 2012; 72: 89-102.

19. Sam-Soto S, Gayón-Vera E. Guía práctica para el abordaje y manejo de lesiones anogenitales por virus de papiloma humano en adolescentes. Acta Pediatr Mex 2006; 27: 151-156.

20. Wiesner C, Cendales R, Murillo R, Piñeros $\mathbf{M}$, Tovar S. Seguimiento de mujeres con anormalidad citológica de cuello uterino, en Colombia. Rev Salud Pública 2010; 12: 1-13.

21. Population Reference Bureau (PRB), Alliance for Cervical Cancer Prevention (ACCP). La prevención del cáncer cervical a nivel mundial. Washington, Estados Unidos: PRB. 2004. Disponible: http://screening.iarc.fr/doc/PRB-ACCP_PreventCervCancer_SP.pdf. Consultado: jun 2016.

22. López-Alegría F, Quezada-Poblete O, SoaresDe Lorenzi D, Sepúlveda-Oyanedel JC. Clinica management of the first ASCUS report in Chile. Prospective single-cohort study. Sao Paulo Med J 2015; 133: 480-487.

23. Hidalgo-Vicario MI, Castellano-Barca G. Virus del papiloma humano y adolescencia. Bol Pediatr 2007; 47: 213-218.

24. Moreno-Altamirano A, López-Moreno S, Corcho-Berdugo A. Principales medidas en epidemiología. Salud Pública Méx 2000; 42: 337-348.

25. Sanabria Negrín JG, Salgueiro Medina VE, Abreu Mérida MB, Lemus Sarracino A, Marrero Fernández $\mathbf{R}$. Incidencia y tendencia de lesiones cervicouterinas en mujeres pinareñas de 10 24 años. 2003-2012. Rev Ciencias Médicas 2014; 18: 4-13. 